\title{
Biologie des saumons de la Nivelle et de l'Adour : renseignements récents obtenus par piégeage dans une passe à poissons
}

Biology relating to salmon in the river Nivelle and the river Adour :
recent information obtained by trapping in a fish ladder

J. Dumas

INRA

St-Pée-sur-Nivelle
A. Marty

Conseil supérieur de la pêche

$\mathrm{Pau}$

La première partie de la communication fait état des renseignements obtenus à partir de léchelle à poissons d'Uxondoa sur le rythme migratoire et la biologie des saumons adultes sauvages et de pisciculture de la Nivelle.

La seconde partie définit les zones de fréquentation actuelle du saumon atlantique dans le bassin de l'Adour, ses caractéristiques biologiques et de son évolution depuis 1950.

The first part of this article supplies the information obtained from the Uxondoa fish ladder about the migratory frequency and biology of wild adult salmon and of the Nivelle pisciculture.

The second part defines the zones currently frequented by the Atlantic salmon in the Adour, its biological characteristics and its evolution since 1950.

\section{Introduction}

Les études exposées dans la présente communication ont débuté en 1977 sous l'impulsion du Plan Saumon, mis en place par le ministère de l'Environnement en 1976.
Elles ont été réalisées par le Laboratoire d'écologie des poissons de l'I.N.R.A. de St-Pée-sur-Nivelle, pour ce qui concerne la Nivelle, et par la délégation régionale $n^{\circ} 7$ du Conseil supérieur de la pêche pour l'Adour et ses constituants. 


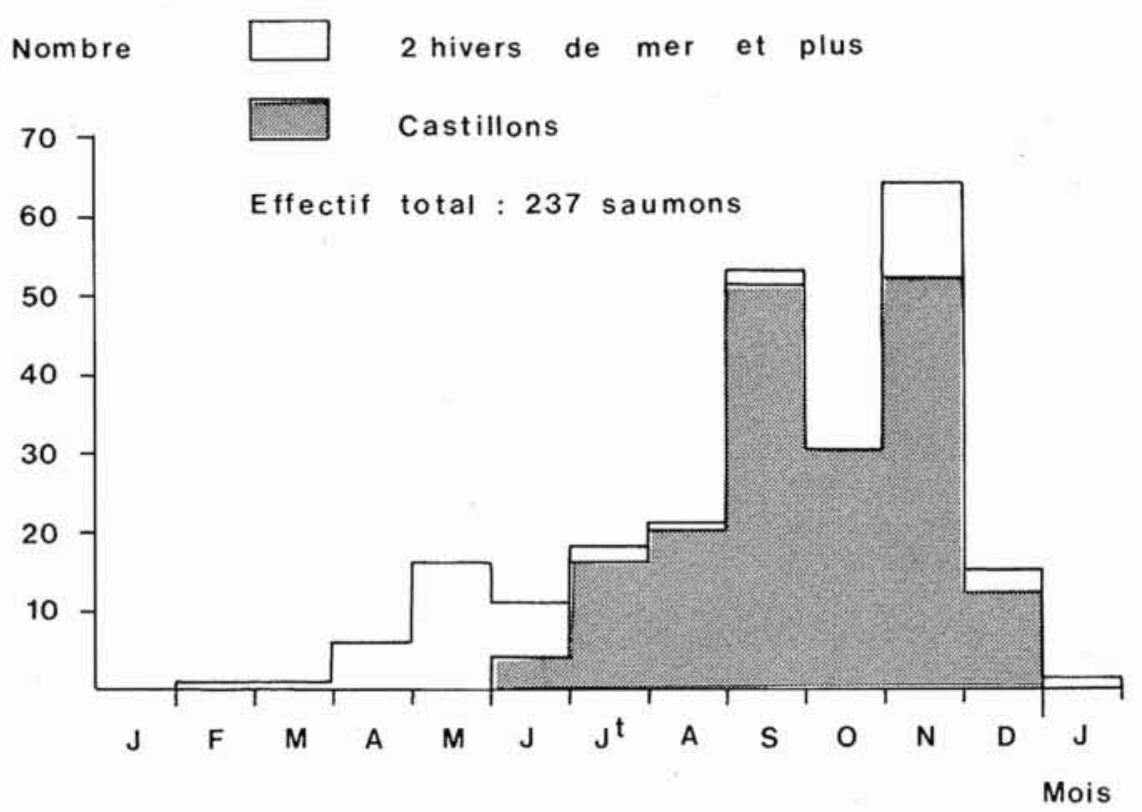

Figure 1. - Fréquence de franchissement de la passe à poissons d'Uxondoa par les saumons adultes sauvages et d'élevage de la Nivelle en 1984 et 1985.

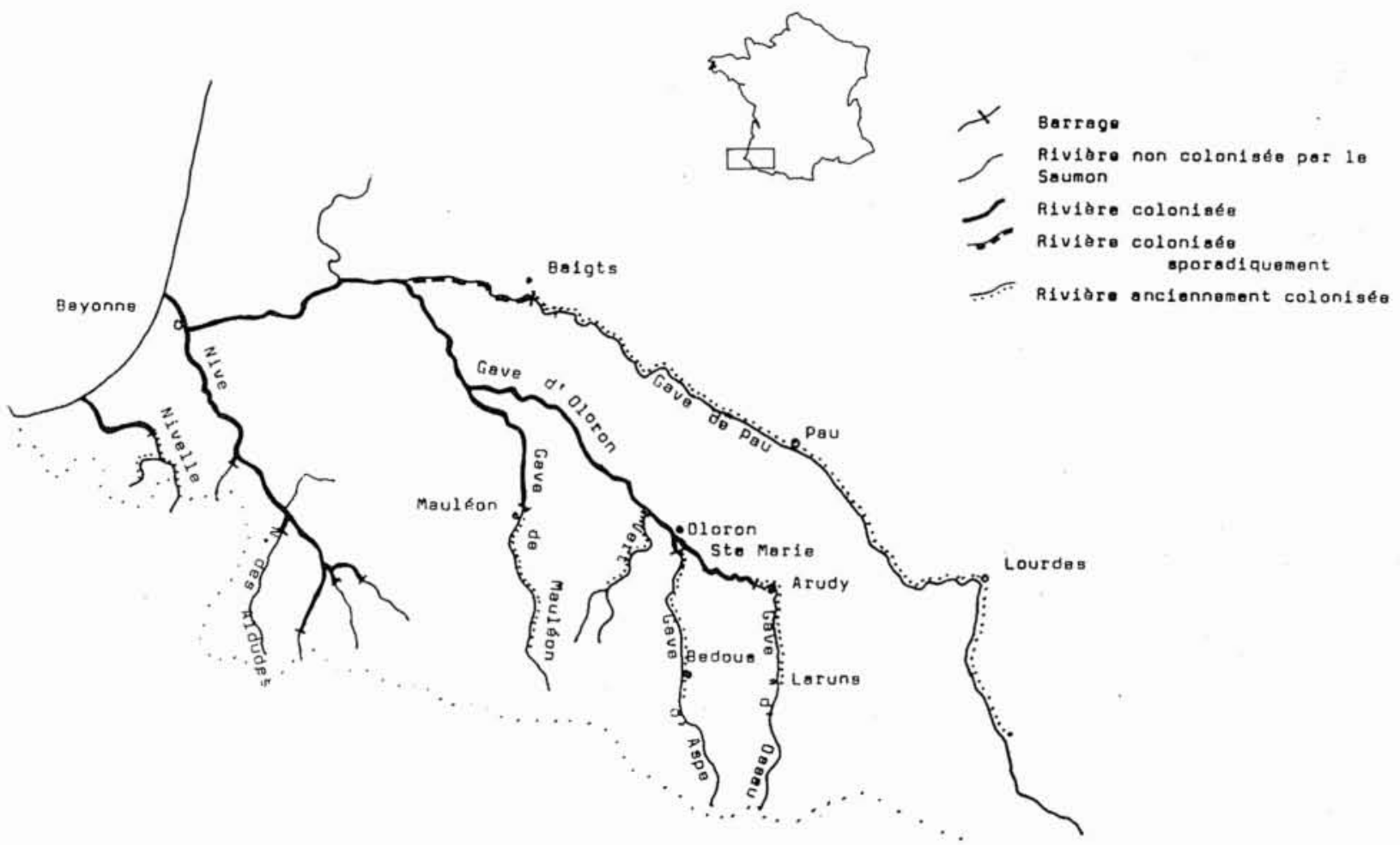

Figure 2. - Répartition du Saumon atlantique dans le bassin de l'Adour et de la Nivelle. 


\section{La population de saumons de la Nivelle (Bilan 1977-1985)}

La Nivelle est un petit fleuve côtier de $38 \mathrm{~km}$ de long des Pyrénées Atlantiques, mais dont seulement $9 \mathrm{~km}$ de cours en eau douce sont accessibles au saumon (fig. 2).

Jusqu'en 1983, des échantillons de pré-reproducteurs ou de reproducteurs sont essentiellement obtenus par pêches électriques d'automne (et accessoirement déclarations de captures par les pêcheurs ou de poissons trouvés morts). Depuis 1984, la mise en service de la passe à poissons d'Uxondoa, munie d'un piège de contrôle, permet des observations et des études plus précises des remontées de saumons, ainsi que d'autres migrateurs de ce cours d'eau. Cet ouvrage expérimental appartient à la Fédération des A.A.P.P. des Pyrénées Atlantiques qui en a délégué la gestion à l'I.N.R.A.

\subsection{La passe à poissons d'Uxondoa}

La station de contrôle est située à $4,5 \mathrm{~km}$ de la limite de salure des eaux sur un barrage d'une hauteur de $2,2 \mathrm{~m}$, difficilement franchissable par le saumon. La passe est constituée de 7 bassins successifs à échancrures latérales (Larinier, 1983) et d'une chambre de capture $\left(12 \mathrm{~m}^{2}\right.$ et $1,5 \mathrm{~m}$ de profondeur) dans sa partie amont. Les poissons ne peuvent s'en échapper. Le débit qui y transite varie selon le niveau d'eau amont de 250 à $850 \mathrm{l} / \mathrm{s}$. Un apport d'eau supplémentaire de $500 \mathrm{l} / \mathrm{s}$ est assuré dans le bassin aval afin d'augmenter le courant d'attrait au pied de l'échelle. Le débit minimum transitant dans ce dispositif est important et correspond au $1 / 10^{c}$ du module annuel de la rivière $\left(7 \mathrm{~m}^{3} / \mathrm{s}\right)$.

Le piège est mis en service de fin février ou début mars à fin décembre ou début janvier et fonctionne de manière continue (nuit et jour), exception faite de courtes périodes de très fortes crues ( 5 jours en 1984 et 7 jours en 1985).

\subsection{Résultats}

Structure et caractéristiques générales de la population de saumons

Un échantillon de 625 saumons est récolté en 9 ans. Il est constitué de $56 \%$ de sujets d'élevage (variation annuelle de 18 à $82 \%$ selon l'effort de repeuplement), issus de saumoneaux de pisciculture libérée cryomarqués; ces derniers sont de deux origines, l'une autochtone de la Nivelle, l'autre écossaise, appelée naturalisée dès la deuxième génération de retour.

Alors que $79,9 \%$ des saumons adultes sauvages n'ont séjourné qu'une année en eau douce avant leur avalaison, la plupart des animaux d'élevage $(57,4 \%)$ ont quitté la Nivelle à l'âge de deux ans.

La majorité des saumons de cette rivière reviennent comme castillons après 1,5 ans de mer $(68,4 \%$ des sauvages, $72,3 \%$ des autochtones d'élevage et $92,6 \%$ des écossais-naturalisés d'élevage). Les autres remontent après 2 ans (petits saumons) et plus rarement 3 ans de vie marine (grands saumons).

La proportion de femelles oscille entre 62 et $66 \%$ selon le groupe d'origine, mais chez les sujets de deux hivers de mer ou plus, elles sont nettement majoritaires avec 82 à
$89 \%$ des effectifs (Dumas et Casaubon, 1985).

A âge marin égal, les caractéristiques métriques des sujets des trois groupes sont assez voisines: de 65,3 à $66,8 \mathrm{~cm}$ (pour des poids de 2,4 à $2,7 \mathrm{~kg}$ en automne) chez les castillons, de 79,3 à $80,7 \mathrm{~cm}$ (pour 4 à $4,4 \mathrm{~kg}$ ) chez les petits saumons, de 93 à $98,6 \mathrm{~cm}$ (pour 6,9 à $8,7 \mathrm{~kg}$ ) chez les grands saumons (Dumas et Casaubon, 1985).

\section{Rythmes de remontées}

Le piégeage sur deux ans de 237 sujets de première remontée permet d'étudier leur comportement, ainsi que le calendrier des retours selon les types d'âges marins (fig. 1).

Ils empruntent l'échelle habituellement aux heures crépusculaires et de nuit $(80,7 \%$ en 1984) et essentiellement en début et fin de crue, parfois pendant la crue si celle-ci n'est pas trop violente $(5$ à $20 \mathrm{~cm}$ d'amplitude de niveau).

Les saumons de 2 et 3 ans de mer peuvent se présenter dès février jusqu'à décembre, voire janvier de l'année suivante, mais ils franchissent plutôt la passe au printemps essentiellement en mai; ceux n'ayant pu se présenter à l'ouvrage avant l'étiage, le franchissent en automne. Les castillons, représentant la majorité des effectifs, remontent de fin juin à fin décembre avec un maximum de miseptembre à mi-novembre.

De mars à mi-juin, période d'ouverture de la pêche au nord de la Dordogne, les saumons de 2 ans de mer et plus représentent la totalité du stock; ils constituent encore $62,3 \%$ des remontées de mars à fin juillet, époque où s'exerce la pêche dans le sud-ouest.

\section{Taux de contrôle des sujets d'élevage}

Tous âges d'eau douce confondus, les poissons marqués d'origine autochtone induisent des taux de capture deux fois et demi plus conséquents que ceux de souche écossaise puis naturalisée : 9,4 contre 3,8 p. 1000 (tableau 1). Les reprises de gros saumons de 2 et 3 hivers de mer sont plus nombreuses chez les premiers $(27,7 \%$ contre $7,4 \%)$; elles sont voisines de celles observées chez les individus sauvages $(31,6 \%)$.

Les saumoneaux de deux ans, compte tenu de leurs plus grandes tailles, induisent des retours de 3 à 5 fois plus importants que ceux d'un an.

Les retours de smolts libérés en 1983 sont vérifiés plus efficacement au moyen du piège; 81,1 et $87 \%$ du stock estimé d'adultes ont été respectivement contrôlés en 1984 et 1985 . Le taux de capture $(32,4$ p. $1000 \mathrm{chez}$ les animaux autochtones et 16,5 p. 1000 chez les écossais-naturalisés) cernent d'assez près le taux réel de retour en Nivelle).

\subsection{Discussion et conlusion}

Les caractéristiques des saumons de la Nivelle sont, pour un même âge marin, très voisines de celles des poissons de l'Adour et du Gave d'Oloron (Marty et Bousquet, 1985).

Bien que constituée d'une majorité de castillons au moment de la reproduction, la population de ce cours d'eau est au printemps exclusivement représentée par des saumons de 2 et plus rarement 3 ans de mer et ces sujets sont encore les plus nombreux jusqu'à la fin de juillet. 


\begin{tabular}{|c|c|c|c|c|c|c|c|c|c|c|c|c|}
\hline \multicolumn{5}{|c|}{ Saumoneaux } & \multicolumn{8}{|c|}{ Saumons adul tes } \\
\hline \multirow{2}{*}{ Origine } & \multirow{2}{*}{$\begin{array}{l}\text { Année de } \\
\text { libération }\end{array}$} & \multirow{2}{*}{$\begin{array}{c}\text { Age } \\
\text { (annêes) }\end{array}$} & \multirow{2}{*}{$\begin{array}{l}\text { Longueur à } \\
\text { la fourche } \\
\qquad(\mathrm{cm})\end{array}$} & \multirow{2}{*}{$\begin{array}{l}\text { Nombre } \\
\text { libęrế }\end{array}$} & \multicolumn{4}{|c|}{ Nombre contrôlé } & \multicolumn{4}{|c|}{$\begin{array}{l}\text { Taux de captures } \\
(p .1000)\end{array}$} \\
\hline & & & & & $1 \mathrm{~h} \cdot \mathrm{m}$ & $2 \mathrm{~h} . \mathrm{m}$ & $3 \mathrm{~h} . \mathrm{m}$ & Total & $1 \mathrm{h.m}$ & $2 \mathrm{htm}$ & $3 \mathrm{h.m}$ & Total \\
\hline \multirow{6}{*}{ Autochtone } & 1979 à 1983 & 1 & 13,4 & 11190 & 48 & 18 & $1^{*}$ & 67 & 4,3 & 1,6 & $0,1^{*}$ & 6,0 \\
\hline & 1980 ã 1983 & 2 & 14,8 & 4459 & 59 & 20 & $2^{*}$ & 81 & 13,2 & 4,5 & $0,5^{*}$ & 18,2 \\
\hline & & Total 1 et 2 & 13,8 & 15649 & 107 & 38 & $3^{*}$ & 148 & 6,8 & 2,4 & $0,2^{*}$ & 9,4 \\
\hline & 1983 & 1 & 14,6 & 151 & 15 & 10 & * & 25 & 99,3 & 66,3 & * & 165,6 \\
\hline & 1983 & 2 & 14,7 & 1576 & 27 & 4 & * & 31 & 17,1 & 2,6 & * & 19,7 \\
\hline & & Total 1 et 2 & 14,7 & 1727 & 42 & 14 & * & 56 & 24,3 & 8,1 & * & 32,4 \\
\hline \multirow{4}{*}{$\begin{array}{l}\text { Ecossaise } \\
\text { puis } \\
\text { naturalisểe }\end{array}$} & 1976 ã 1982 & 1 & 13,1 & 33296 & 64 & 1 & & 65 & 1,9 & 0,03 & & 1,9 \\
\hline & 1976 ã 1983 & 2 & 15,3 & 9366 & 86 & 11 & * & 97 & 9,2 & 1,2 & * & 10,4 \\
\hline & & Total 1 et 2 & 13,6 & 42662 & 150 & 12 & * & 162 & 3,5 & 0,3 & * & 3,8 \\
\hline & 1983 & 2 & 15,9 & 4175 & 59 & 10 & * & 69 & 14,1 & 2,4 & * & 16,5 \\
\hline
\end{tabular}

Tableau 1. - Contrôles (de 1977 à 1985) de saumons adultes cryomarqués, libérés en Nivelle comme saumoneaux d'élevage (de 1976 à 1983). Deux origines sont comparées : autochtone et écossaise de la Thurso, appelée naturalisée dès la $2^{\circ}$ génération de retour en Nivelle. Les retours des lots libérés avant 1983 sont pour la plupart contrôlés par pêche électrique d'automne, tandis que ceux des lots libérés en 1983 le sont par piégeage et font l'objet d'une comptabilité séparée.

Tableau 2. - Âges et longueurs des smolts de saumon atlantique du bassin de l'Adour, lors de différentes dévalaisons printanières.

\begin{tabular}{|c|c|c|c|c|c|c|c|c|c|c|c|c|c|c|c|c|c|c|}
\hline \multirow{2}{*}{\multicolumn{2}{|c|}{$\begin{array}{l}\text { Astac( }(S) \\
\text { Et }\end{array}$}} & \multirow{3}{*}{$\begin{array}{l}\text { DATES } \\
\text { DE } \\
\text { CAPTURES }\end{array}$} & \multirow{3}{*}{ ECHANTILLOL: } & \multirow{2}{*}{\multicolumn{3}{|c|}{$\begin{array}{l}\text { RCPAATITION PAR } \\
\text { CLASSE D'ACE }(x)\end{array}$}} & \multicolumn{12}{|c|}{ LONGUEUA MINIMALE, ROYENNE ET mAXIMALE on $\mathrm{cm}$} \\
\hline & & & & & & & \multicolumn{3}{|c|}{ POPULATIOH TOTALE } & \multicolumn{3}{|c|}{1 an } & \multicolumn{3}{|c|}{2 ans } & \multicolumn{3}{|c|}{3 ons } \\
\hline tITU DE & CAP TUAT & & & 1 an & 2 ang & 3 ans & min. & moy. & $\max$. & $\min$. & may, & $\max$. & $\sin$. & moy. & $\max$. & min. & noy, & $\max$. \\
\hline $1942-88$ & Anก:PP-CAVE & & 1.022 adultes & 71,0 & 28,0 & 1,0 & & & & & & & & & & & & \\
\hline $194 K-47-\Delta A$ & CAVI D'OLORON & & 492 molts & 57,5 & 42,3 & 0,21 & & 16,1 & & & 15,6 & & & 16,9 & & & 20,4 & \\
\hline 1969 & GAVE DIOLOHON & $13 / 3-18 / 4$ & $1.821 \mathrm{mmoltg}$ & 67,4 & $25,:$ & 7,5 & 14 & 19,5 & 28 & 14 & 19,4 & 21 & 18 & 21,5 & 23 & 21 & 23,2 & 27 \\
\hline 1970 & CAVF DIOLORON & $1 / 4-22 / 4$ & 2.945 geoles & 80,0 & 12,0 & 8,0 & 14 & 10,3 & 27 & 14 & 17,7 & 20 & 18 & 21,5 & 23 & 20 & $\underline{22,4}$ & 26 \\
\hline 1971 & EAVE NPOLOAON & $20 / 7-20 / 4$ & 1.677 onolts, & 43.2 & 45,0 & 11,8 & 15 & 20,7 & 27 & 15 & 19,7 & 21 & 1a & $\underline{20,8}$ & 24 & 20 & $\underline{23,6}$ & 26 \\
\hline 1972 & GAUF DOOLOROR: & $10, / 4-20 / 4$ & 218 sacolts & 55,2 & 38,0 & 6,8 & 17 & 21,9 & 28 & 17 & 21,3 & 23 & 20 & 22,1 & 26 & 23 & $\underline{26,0}$ & 27 \\
\hline 1971 & CAVI O.OLORON & $5 / 4-25 / 4$ & 1.114 enolth & 57,9 & 27,1 & 5,0 & 15 & 19,3 & 26 & 15 & 19,3 & 21 & 18 & 21,0 & 22 & 20 & $\underline{22,6}$ & 25 \\
\hline 1978 & CaUS VOLORDN & $20: 3=5 / 5$ & 695 moll, & 69 & 28 & ? & 14 & $\cdot 9,5$ & 27 & 14 & 18,4 & 21 & 17 & $\underline{21,5}$ & 26 & 19 & $\underline{23,1}$ & 27 \\
\hline 1960 & Nive & $2 h / 1-2 A / 4$ & 714 molts & 95,7 & 4,3 & 0 & 14 & 19,8 & 26 & 14 & 19,0 & 24 & 22 & 24,2 & 26 & & & \\
\hline 1981 & NIVI & $w / 2-19 / 4$ & $1.100=0.01: 9$ & 98,3 & $1, ?$ & 0 & 14 & 10,4 & 25 & 14 & 19,3 & 24 & 20 & $22, ?$ & 25 & & & \\
\hline 1982 & Nivt & $10,-27$. & 1.450 nectes & 94,1 & 5.7 & 0 & 13 & 17,9 & 26 & 13 & 17,6 & 22 & 19 & $\underline{22.1}$ & 26 & & & \\
\hline
\end{tabular}


Pendant la période d'exercice de la pêche, la structure de son stock parait peu différente de celle observée dans les rivières du massif Armoricain (Baglinière et al., 1985) ou dans l'Adour-Gave (Marty et Bousquet, 1985) où les petits et grands saumons sont également les plus nombreux à cette époque.

Ces deux années de piégeage mettent en évidence la nécessité d'une meilleure adaptation de la période d'ouverture de la pêche au stock en présence dans la Nivelle; actuellement pratiquée de mars à juillet, elle s'exerce sur des saumons de printemps peu nombreux alors que les castillons revenant principalement en début d'automne sont très peu exploités.

Les repeuplements avec la souche locale, dont les saumoneaux procurent les meilleurs taux de recapture et une bonne proportion de poissons de printemps, sont à promouvoir afin d'obtenir une production optimale d'adultes en retour.

Le piège de contrôle d'Uxondoa, très performant, s'avère un outil de connaissance indispensable qui permet non seulement de comptabiliser et de caractériser les stocks de migrateurs, mais aussi de les gérer (ex : capture de géniteurs pour reproduction artificielle ou déplacement d'adultes dans des zones non accessibles).

\section{La population de saumon du bassin de l'Adour (Bilan 1977-1983)}

Les principaux constituants du bassin de l'Adour fréquentés par le saumon sont ceux des sous-bassins de la Nive et du Gave d'Oloron. Ils sont situés entre le $43^{\circ}$ et le $44^{\circ}$ degré de latitude nord et ont une vocation salmonicole marquée.

Pourtant, les captures de saumons ont fortement baissé par rapport au début du siècle pour se stabiliser à l'heure actuelle à un très bas niveau (pêche à la ligne et pêche professionnelle confondues, environ 1000 captures en moyenne par an).

L'une des causes premières de cette régression des saumons est l'édification - ou le rehaussement - de nombreux barrages qui ont réduit de plus de $50 \%$ l'aire de répartition du saumon dans le bassin de l'Adour (fig. 2).

\subsection{Caractéristiques des saumons du bassin de l'Adour}

\subsubsection{Les juvéniles}

Ils ont été étudiés à partir d'inventaires piscicoles réalisés en période d'étiage sur la Nive et le Gave d'Oloron.

\section{Le stade tacon}

Les densités rencontrées sur le Gave d'Oloron et la Nive sont, le plus souvent, de l'ordre d'une centaine d'individus par hectare de radier (maximum : 1000 ind/ha de radier).

Les jeunes saumons sont âgés de $\mathrm{O}+$ an (taille moyenne : $11-12 \mathrm{~cm}$ ), $1+$ an (taille moyenne : $16-18 \mathrm{~cm}$ ) et, plus rarement, $2+$ ans (taille moyenne : $22-24 \mathrm{~cm}$ ).

Par rapport aux autres bassins français, les densités observées sur le bassin de l'Adour sont faibles mais les performances de croissance sont remarquablement élevées.

\section{Le stade smolt (tableau 2)}

Les smolts ont été étudiés au cours des dévalaisons printanières sur le Gave d'Oloron (Bousquet, 1979) et sur la Nive (Bousquet, 1983).

Sur les deux rivières, on constate que la migration s'étale de mars à mai avec un maximum d'intensité autour de la mi-avril.

Sur le Gave d'Oloron, la population de smolts est composée à $60-70 \%$ de poissons âgés de $1+$ an, à $25-35 \%$ de poissons âgés de $2+$ ans et à $1-5 \%$ de poissons âgés de $3+$ ans.

L'âge moyen des smolts de la Nive est moins élevé : 90 à $95 \%$ de $1+$ an, 5 à $10 \%$ de $2+$ ans, absence de $3+$ ans.

Sur les deux cours d'eau, les tailles (longueur totale) s'échelonnent de 13 à $27 \mathrm{~cm}$ pour des poids allant de 18 à $165 \mathrm{~g}$.

Les smolts du Gave d'Oloron avaient déjà fait l'objet d'une étude spécifique, il y a quelques 40 années (Vibert, 1950). La comparaison des résultats d'alors avec ceux d'aujourd'hui amène à faire les constats suivants :

- même période de dévalaison, même structure d'âge des smolts:

- à l'heure actuelle, tailles et poids sensiblement plus élevés (longueur moyenne augmentée de $20 \%$; poids moyen augmenté de $46 \%$ ) mais nombre d'individus dévalants probablement beaucoup plus faibles.

\subsubsection{Les adultes}

1024 échantillons d'écailles de saumons adultes provenant du bassin de l'Adour ont été récoltés entre 1977 et 1981. Cet échantillonnage s'est déroulé du 20 février au 31 juillet pour les pêcheurs aux filets en zone maritime et du début mars au 31 juillet pour les pêcheurs à la ligne et aux engins en zone fluviale.

\section{Résultats :}

- pour la vie en eau douce :

- $72 \%$ des saumons y ont passé 1 an

- $28 \%$ des saumons y ont passé 2 ans

- moins de $1 \%$ y ont passé 3 ans.

Ces résultats ne coïncident pas exactement avec ceux observés sur les smolts en cours d'avalaison de 1978 à 1983.

- pour la vie en eau de mer:

la quasi-totalité des saumons capturés y ont passé 1 à 3 ans (tableau 3), exceptionnellement 4 ans ( $1 \% 0)$.

- Les saumons de 3 ans de mer représentent une proportion de $19 \%$

- Les saumons de 2 ans de mer représentent une proportion de $75 \%$

- Les saumons de 1 an de mer représentent une proportion de $6 \%$.

Ces proportions fluctuent annuellement de façon important suivant les différents groupes d'âges (tableau 4).

Les longueurs totales des poissons capturés varient de 56 à $106 \mathrm{~cm}$ et $90 \%$ des prises sont comprises entre 70 et $90 \mathrm{~cm}$. Les poids fluctuent de 1,97 à $11,4 \mathrm{~kg}$ et plus de $90 \%$ des captures pèsent de 3,7 à $6,7 \mathrm{~kg}$. 


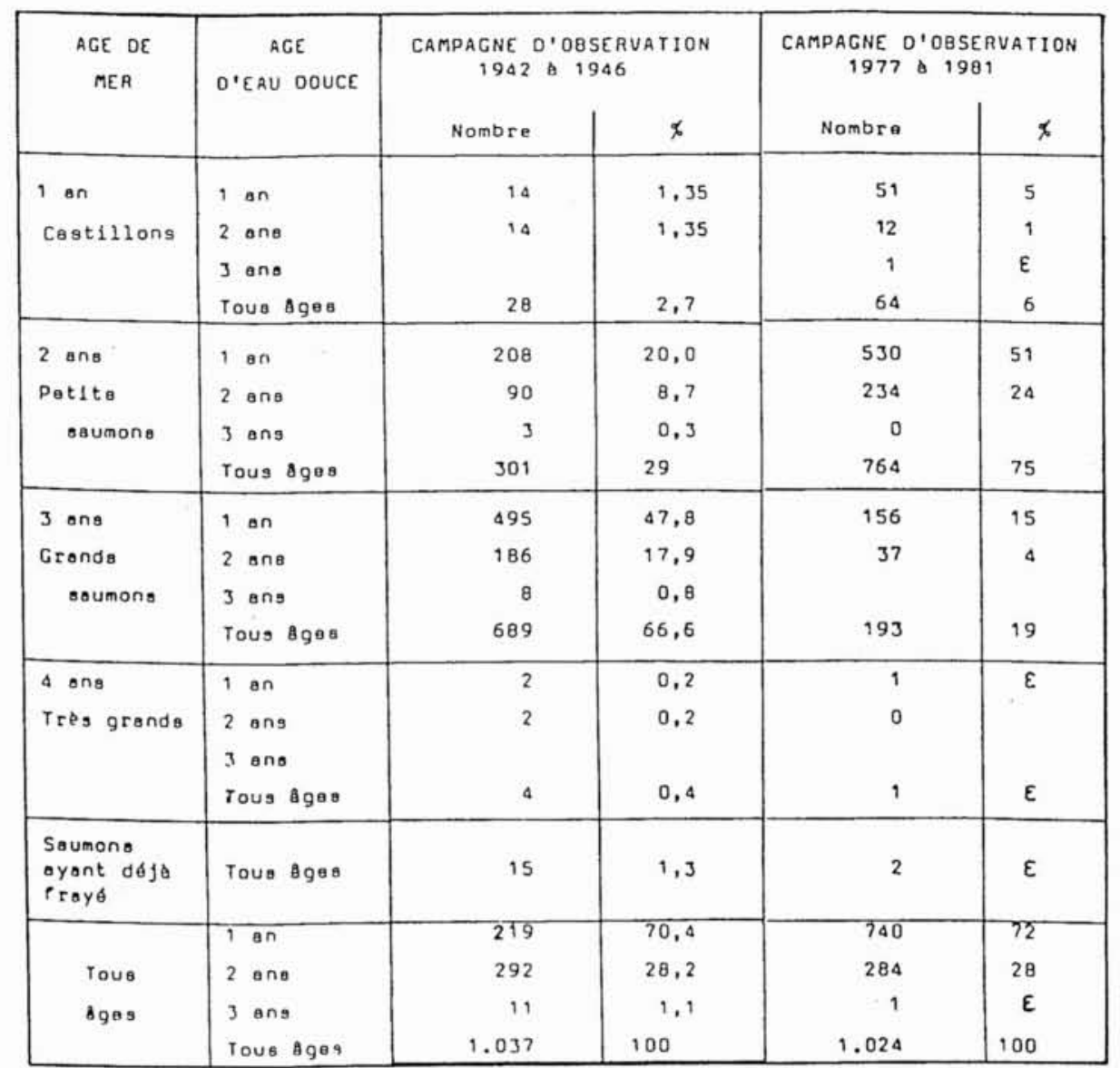

Tableau 3. - Structure d'âge des populations de Saumon de I'Adour (C.S.P., 1985; Vibert, 1950)

Tableau 4. - Différents types de saumons capturés par les filets et les pêcheurs à la ligne de 1977 à 1981.

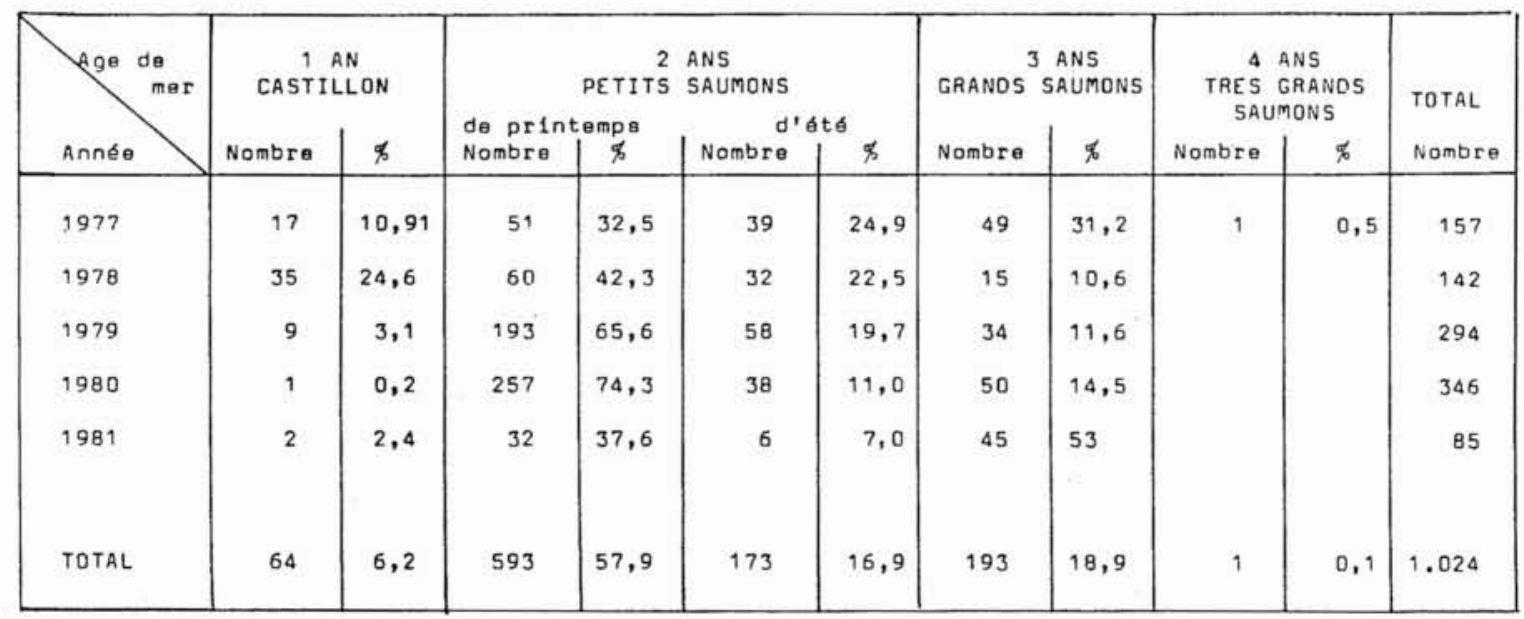


Les résultats moyens par groupe d'âge de mer sont les suivants :

- castillons : $65,6 \mathrm{~cm}$ pour $2,9 \mathrm{~kg}$

- petits saumons ( 2 et $2+$ ) : $80,2 \mathrm{~cm}$ pour $5,0 \mathrm{~kg}$

- grands saumons : $94,2 \mathrm{~cm}$ pour $7,9 \mathrm{~kg}$

\subsection{Discussion - Conclusion}

Les saumons de l'Adour étudiés se caractérisent actuellement par :

- une relative homogénéité des caractéristiques (majorité de 2 ans de mer),

- une structure d'âge de rivière peu étalée ( 2 ans), liée à une bonne croissance.

On remarque par ailleurs qu'une migration de saumons se recrute au total sur 5 (exceptionnement 6 ) ans avec :
- $95 \%$ du stock qui ne relève que de 3 années de fraie - plus de $50 \%$ du stock ne relève que d'une seule année de fraie.

Les saumons de 2 ans de mer constituent actuellement la grande majorité d'une cohorte de remontée et indiquent de ce fait une très profonde modification de la durée de vie des saumons en mer par rapport aux années 1942-1946 (Vibert, 1950).

Cette modification est marquée par une diminution du temps passé en mer et a certainement pour origine les caractéristiques actuelles d'exploitation qui s'exercent préférentiellement sur certaines classes d'âges à la différence de ce qui se passait autrefois.

Il y a par ailleurs lieu de penser que les proportions de petits saumons d'été et de castillons sont sous-estimées dans cette étude du fait que la campagne d'échantillonnages ne s'est effectuée que du mois de mars à fin juillet.

\section{Conclusion générale}

Les études poursuivies sur les populations de saumon du sud-ouest de la France, dont un aperçu a été donné dans les communications qui précèdent, ont notamment pour aboutissement de définir une forme de gestion permettant d'optimiser l'exploitation de la ressource.

Une composante essentielle du problème réside dans la connaissance de l'état des stocks et de leur dynamique.

Sur le bassin de l'Adour, système hydrographique de grande étendue, à fort débit et crues importantes, la mise en place d'un piège de capture en zone aval pose de gros problèmes techniques - et financiers - qui n'ont pas été résolus à ce jour. En l'absence de stations de piégeage, une solution de remplacement, pour connaître les stocks, a consisté en enquêtes auprès des pêcheurs, professionnels et amateurs, et collecte des renseignements sur les captures. Le défaut majeur d'une telle approche est évident : les données recueillies ne portent que sur une fraction de l'année (la période durant laquelle la pêche est autorisée, soit mars à juillet) ce qui, compte tenu de la biologie de l'espèce, induit un biais d'échantillonnage important.

La méthode actuelle d'étude du stock du saumon de la Nivelle, basée sur l'utilisation d'un piège, permet le contrôle systématique de tous les saumons adultes, et ceci tout au long de l'année. Cette technique permet bien évidemment de mieux cerner la réalité des migrations sous différents aspects (effectifs, rythme, contrôle des taux de retour), mais aussi de participer à la gestion de la ressource (captures de géniteurs pour pisciculture, définition de quota...).

C'est ainsi par exemple qu'à pu être mis en évidence sur ce cours d'eau une importante remontée très tardive de castillons qui devrait permettre de repenser les périodes d'ouverture de la pêche du saumon dans cette rivière.

Il apparaît ainsi que les systèmes échelles à poissons + pièges de captures constituent un outil de choix pour la mise en place d'un plan de gestion prenant en compte des réalités biologiques.

\section{Bibliographie}

Bagliniere J.L., Prouzet P., Porcher J.P., Nihouarn A., Maisse G., 1985. - Caractéristiques générales des populations de saumon atlantique (Salmo salar $L$.) des rivières du Massif Armoricain*.

BOUSQUET B., 1979. - Biologie et migration des smolts de Saumon atlantique (Salmo salar L.) dans les bassins Loire Allier et Adour - Gave d'Oloron. Thèse $3^{c}$ cycle Biol. Anim. I.N.P. Toulouse 67 p. BOUSQUET B., 1983. - Situation du Saumon atlantique dans le bassin de l'Adour - Etude du stade smolt sur la rivière Nive lors des dévalaisons printanières 1980, 1981 et 1982. C.S.P. 31 p.

Dumas J., Casaubon J., 1985, - Etude, restauration et gestion de la population de saumon atlantique (Salmo salar $L$.) de la Nivelle (Pyrénées Atlantiques)*

LARINIER M., 1983. - Guide pour la conception des dispositifs de franchissement des barrages pour les poissons migrateurs. Bull. Fr. Piscic., $\mathrm{n}^{\circ}$ spécial, $59 \mathrm{p}$.

MARTY A., BousQUeT B., 1985. - Le point sur les connaissances des populations naturelles de saumons du bassin de l'Adour*.

VIBERT R., 1950. - Recherches sur le Saumon de l'Adour (Salmo salar L.) (Ages, croissance, cycle génétique, races) 1942-1948. Ann. St Cent. Hydro. appl., 3, 27-148.

- In Thibault M. et Billard (eds.) « La restauration des rivières à saumons». Colloque franco-québécois, mai-juin 1985, Bergerac. INRA Publ., Paris (sous presse). 

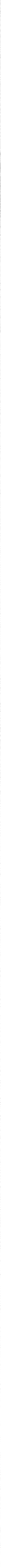